\title{
Athletic Training Analysis System Research based on Physiological Calculation
}

\author{
Ding Xiong ${ }^{1}$ and $\mathrm{Lu} \mathrm{Yan}^{2}$ \\ 1, 2, School of Information Science \& Engineering \\ Hunan International Economics University \\ Changsha, China, postcode: 410205 \\ ${ }^{1,2}$ E-mail:66754252@qq.com
}

\begin{abstract}
The physiological state data is the more important on the athletes' aided training and competition. Physiological Plan system is designed and implemented in this study, the system is divided into hardware layer, data processing layer, algorithm layer and interface layer. The hardware of Physiological Plan is based on the Berkeley Tricorder platform, data processing level is aiming to transform kinds of sensors to a uniform format, algorithm level includes algorithms of filter, peak finder, outlier detection, etc, coaches interact with the system through the interface level. Physiological Plan can collect ECG, EMG, bioimpedance, 3-axis accelerations of the player, real-time feedback of the data is provided to coaches, and the physiological states of the player are monitored and analyzed to help them. The experiment shows that Physiological Plan is valuable for coaches to monitor and it analyze the states of players.
\end{abstract}

Keywords: Human computer interaction, Physiological computing, Electrocardiogram (ECG), Electromyogram (EMG), Auxiliary training

\section{Introduction}

Under physiological computing (Physiological Computing) mode [1], the user does not need to take the initiative to enter the interactive information, the physiological state of the user can be collected and analyzed in real time through the physiological sensing devices of system, and these states are converted into the control input, and feedback is provided the user. Physiological Computing provides a new way for the perception of the user's physiological state and intention, if opens up new research directions for humancomputer interaction, but it also brings a number of challenges. Physiological computing technology can be used in a practical system, the following three key issues must be solved [2]:

(1) physiological psychology theory and practical application;

(2) physiological signal processing algorithms;

(3) physiological computing software tools.

How the physiological states of the user are collected and analyzed? How they are used interactive system? These is a hot issue for current research.

The commonly used signals of physical calculation include EMG, ECG, blood oxygen saturation and skin impedance. The EMG can be used to perceive the muscle [3], and to identify the finger activity [4] and to perceive the degree of user happy [5]. ECG signal is influenced by physical activity and mental stimulation [6], it can be used to control the game [7] and to understand the psychological state [8].

Physiological computing has made some important progress in the aspect of system development. Björn Hartmann developed the Exemplar to help users in a sensor interaction [9]. The model programming method is used in Exemplar, it supports image programming, it is convenient to man-machine interaction of non machine learning 
experts. Similarly, Daniel Ashbrook developed a gesture design tool MAGIC based on action of the sensor [10], the dynamic time warping (DTW) is used as a gesture recognition algorithm, designers are helped faster and more convenient to use the interaction based on sensor device.

Car Coach project provide computer aided adaptive based on user driven behavior modeling [11]; Human Dynamics research team has developed a set of known as the "I Sensed" system(http: //cba.mit.edu/events/02.01.retreat/ Pentland.ppt), a set of wearable devices is used in the system, which contain a variety of sensors, the daily behavior is recorded, and then the analysis and modeling of the sensor data are done, and it to be able to predict the user's daily behavior. Carnegie Mellon University's human computer interaction Institute researchers studied how the computer system can identify the user state like people, the user is interrupted appropriately in a natural way[12]. The multichannel sensing technology is used based on sensor when the user's attentionis obtained. Under the guidance of Gregory D.Abowd professor in Georgia Institute of technology, the psychology data modeling of mentally retarded children and auxiliary medical issues are investigated. Abaris is a therapy system for supporting clinicians in autistic children psychological intervention [13].

Marc Bächlin and KilianFörster in ETH wearable computing laboratory developed a set of swimming wearable assistant system Swim-Master [14]. The athlete's speed, arm and leg movement, rotation can be monitored real-time in the system. The information can also be feedbacked to coaches to evaluate the quality of movement. Witilt researched the fitness area at Berkeley University of California, three-dimensional accelerometer and gyroscope are used in this study to analyze and perceive users' upper limb and body movements, the main action of user fitness can be identified, and the energy consumption is calculated for the user fitness [15]. Florian and Bernt designed a system with wearable sensors and video, skiers are helped in skiing movement perception, and then athletes are helped understand the advantages and disadvantages of their own [16]. Michael and Bernhard developed a football Interactive system, the key action can be acquired understanded in the football match, the coaches are helped for effective analysis of competition and player' state [17]. The objective of study was that transform kinds of sensors to a uniform format; algorithm level includes algorithms of filter, peak finder, outlier detection, etc; coaches interact with the system through the interface level

\section{Materials and Methods}

\subsection{The Overall Framework}

Physiological Plan is designed and implemented, the system may be divided into four main layers: the hardware layer, the data processing layer, algorithm layer and interface layer. Figure 1 is a framework diagram of Physiological Plan system. 


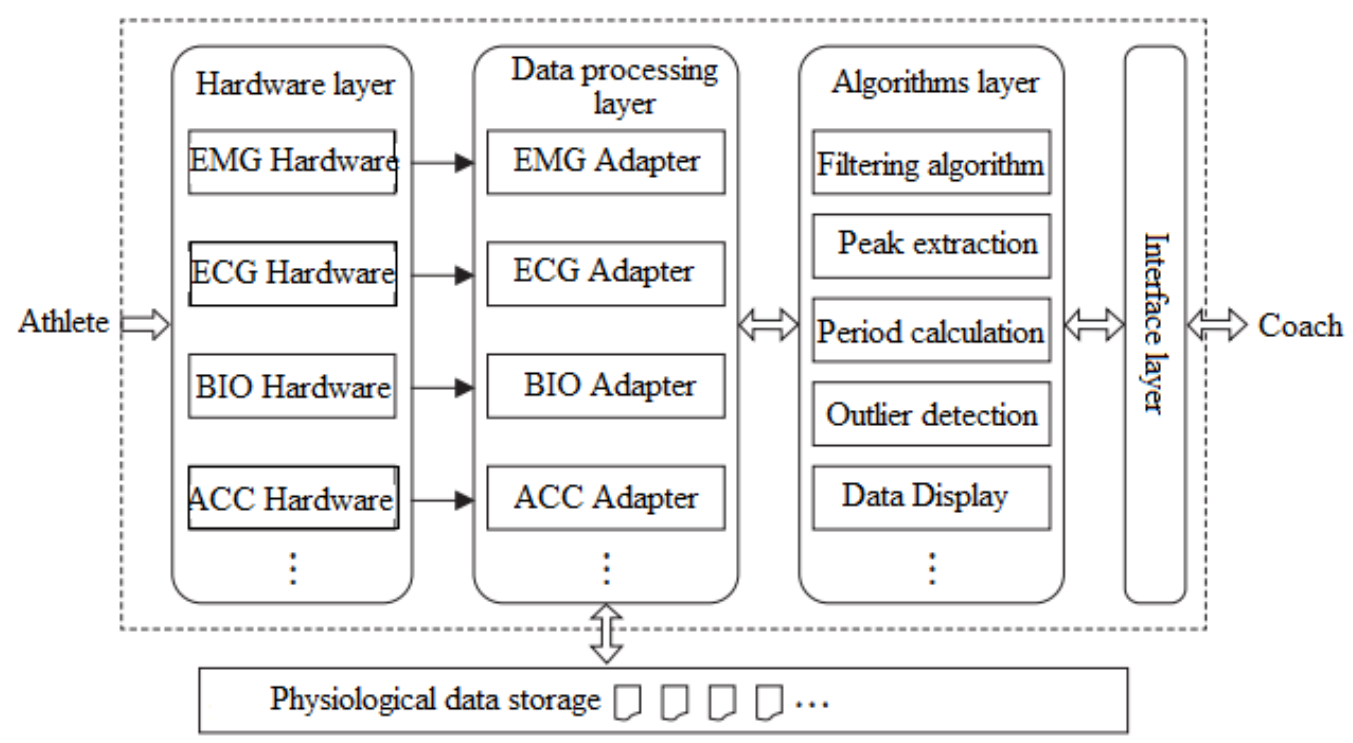

Figure 1. Framework Diagram of Physiological Plan System

Hardware layer include various sensor devices, these devices are responsible for realtime acquisition of athletes' physiological signals, such as muscle electrical signals (EMG), electrocardiograph (ECG), bioelectrical impedance (BIO), Accelerated motion information (ACC). An integrated high physiological testing equipment Berkeley Tricorder is used to complete the data collection [18]. Because of the data formats of different hardware is different, data in different formats need to be sent to data processing layer for unified data format. Adapter is designed in data processing layer for different sensor. Each adapter is used for a specific type of signal acquisition device, such as EMG adapter is corresponding to a variety of muscle signal acquisition equipment, ECG adapter is corresponding to a variety of ECG acquisition equipment. Each adapter function is that data of sensor is converted into the unified format data, so as to facilitate post-processing and display of data, it is also responsible for storing and reading the related physiological data. After the data are captured in hardware layer and they are processed in process layer, these data $\backslash$ will be analyzed and understanded by the algorithm layer. Algorithm layer in the order includes filtering algorithms, the peak extraction algorithm, cycle calculation algorithm, Outlier detection algorithm and data display. After five data processing section, data will be handed over to the user in interface layer. Main contents of the interface layer comprises two parts. On the one hand, Physiological state of the athletes is a real-time display to the coach, on the other hand, the athletes are interaction with coaches, athlete accepts coach input information, and these information are converted into interactive tasks which are passed to the algorithm layer or data processing layer, and the final results are returned to the user.

\subsection{Hardware Layer}

Berkeley Tricorder is used in Physiological Plan hardware and Berkeley Tricorder is developed the BID Laboratory at Berkeley California University [18]. Berkeley Tricorder is a physiological signal acquisition platform, electrocardiogram (ECG), electromyography (EMG), bio impedance (Biolmpedance), oxygen saturation (Pulse Oximetry) and the three axis acceleration can be measured for user. The electrocardiogram (ECG), electromyogram (EMG), bio impedance (BioImpedance) can reflect the athlete's mental state, three axis acceleration can capture athlete's motion state. Berkeley Tricorder communicates with the tablet PC through Bluetooth devices, and it can obtain signal of players on the court at any point. 
Berkeley Tricorder via Bluetooth devices takes communication with tablet computer, the Bluetooth adapter is used in Jabra A320s (http: //www.jabra.com/Sites/Jabra/Naus/Headsets/Pages/ JabraA320s.aspx), the transmission distance of the adapter is up to $100 \mathrm{~m}$, and the site size of badminton doubles is $13.4 \mathrm{~m} * 6.10 \mathrm{~m}$, so the system is not affected by the players in the field location restrictions, this device can capture athlete's signal in arbitrary point of the field.

\subsection{Data Processing Layer}

Data processing layer is the unified data format for various sensors to facilitate later processing. System data input types include electrocardiogram (ECG), electromyogram (EMG), bio impedance (BioImpedance) and the three axis acceleration.

The binary file record is used in the Tricorder raw data. The file is divided into contiguous blocks, each block is 512 bytes, the first two bytes in block indicates whether the device is reset, there are 3 bytes per group in the remaining 510 bytes, the first byte in each group is data type, last two bytes of data value are the high bit and the low bit. The format is shown in Table 1. The definition of data types is shown in Table 2.

Table 1. Tricorder Original Data File Format

\begin{tabular}{llll}
\hline Byte & Content & Byte & Content \\
\hline $1 \sim 2$ & Restart marker & 8 & Data low bit \\
3 & Data type 1 & $\ldots$ & $\ldots$ \\
4 & Data high bit & 510 & Data type n \\
5 & Data low bit & 511 & Data high bit \\
6 & Data type 2 & 512 & Data low bit \\
7 & Data high bit & & \\
\hline
\end{tabular}

Table 2. Tricorder Data Types

\begin{tabular}{llll}
\hline Type & Species & Type & Species \\
\hline 0 & EMG & 4 & Z axis acceleration \\
1 & ECG & 19 & Bioimpedance real part \\
3 & X axis acceleration & 20 & Bioimpedance imaginary part \\
4 & Y axis acceleration & & \\
\hline
\end{tabular}

In order to ensure the real-time and accurate transmission frequency, the transmission frequency can not be too low between Tricorder data and the computer, otherwise the captured data will be insufficient, error is relatively large; at the same time, the transmission frequency can not be too high, otherwise the computer processing is not timely, there may be delay. After testing, the transmission frequency of each sensor is finally selected and is shown in Table 3.

Table 3. Data Transmission Frequency

\begin{tabular}{ccccc}
\hline Type & EMG & ECG & $\mathrm{n}^{\text {Acceleratio }}$ & \multicolumn{2}{c}{ ce } \\
\hline $\begin{array}{c}\text { Frequency/ } \\
\mathrm{Hz}\end{array}$ & 256 & 256 & 256 & 256 \\
\hline
\end{tabular}

In order to make the unified data format for each sensor, data processing layer uses the adapter pattern, each kind of sensor corresponds to an adapter. The original data is represented by the integer array, the data structure after the adapter conversion includes four contents: category, time, value and expansion. Class represents the sensor data types, 
such as ECG, EMG etc., the time label ensures that the data still retain the original time information after noise processing and post-processing, so as to calculate the value of the sensor changes with the time, the expand content is no use. The adapter has transformed input and output format, and it has taken into account the data processing functions.

\subsection{The Algorithm Level}

Physiological Plan algorithms layers include filter, peak extraction, cycle calculation, Outlier detection and data display, the major of data display is an ordinary file and data manipulation.

\subsubsection{Filtering Algorithm}

Common filtering algorithm has a finite length unit impulse response (FIR) filter ( $h t t p$ : /len.wikipedia.org/wiki/FIR_filter), Fast Fourier Transform (FFT) filter (http: //en.wikipedia.org / wiki / Filter_bank\# FFT_filter_banks) and so on. In order to select a relatively better performance of the filter, there are a total of 22 ECG data in the actual collection of 12 players, their filtering effect of different combinations are analyed and compared by the FIR filtering and FFT algorithm, and the results are shown in Figure 2.

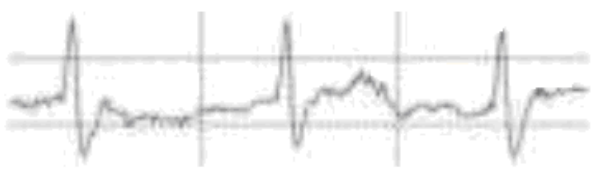

(a) Original waveform

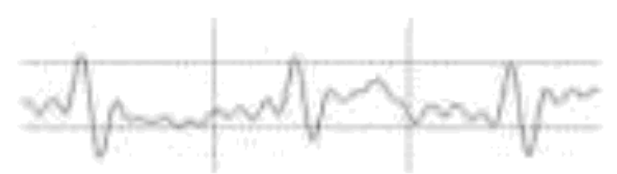

(b) FFT filter

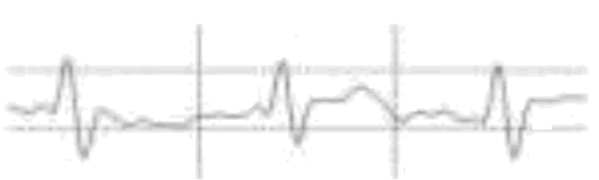

(c) FIR filter

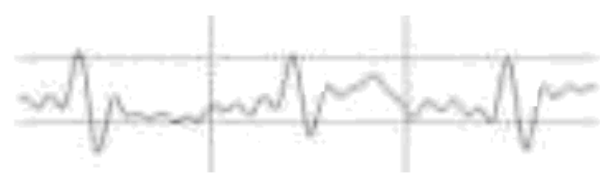

(d) First using FFT filter, then to use FIR filter

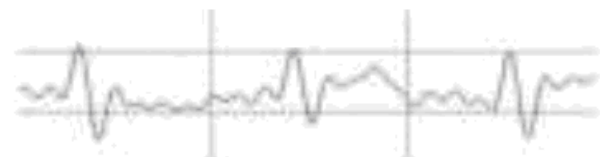

(e) First using FIR filter, then to use FFT filter

Figure 2. The Electrocardiogram (ECG) Signal Filtering Effect with FIR Filtering, FFT Filtering and Their Combinations

Judging from the waveform, the effect of the FIR filter is preferably, FIR filter can eliminates random noise in the original data, a very good smoothing effect has been played, the end result inf the experimental results is better than other filters. Although FFT filter ensures balance, some small ripples are left after processing. Combination with FFT and FIR filters has similar effect with alone FFT. FIR filter is chosen as the original data.

\subsubsection{Peak Extraction Algorithm}

After filtering the raw data, apex is extracted by the peak algorithm. Peak extraction algorithm is developed based on Julius O.Smith findpeaks tools. The algorithm assumes that the peak is a zero-crossing point in the first derivative of the waveform, and its slope exceeds a user-defined threshold. Figure 3 is findpeaks renderings. Figure 3 (a) is the original data, Figure 3 (b) is the vertex extraction results, which is marked with numbers. 


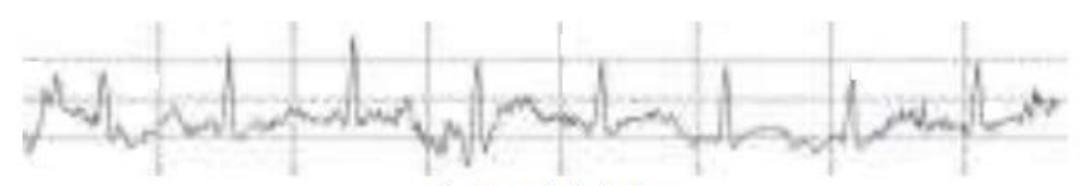

(a) Original data

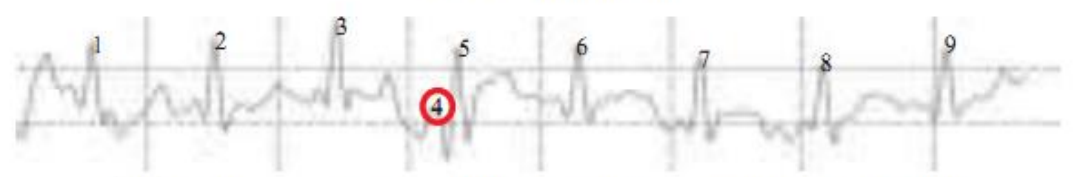

(b) Vertex extraction results, the results are marked with numbers

Figure 3. Peak Extraction Algorithm Results

Figure 3 (b) shows that basic vertex can be extracted by find peaks. the eight vertices are correctly extracted out in Figure 3 (a). However, due to original data inevitably exists noise, the extraction findpeaks are affected, e.g. the vertex of serial No. 4 is not the peak in fact(which is marked in red circle in Figure 3 (b)), but the data is caused by the interference, and the extraction error is made. To address this problem, Outlier detection algorithm is used to remove these false peaks.

\subsection{Period Calculation}

The role of the cycle calculation is that the cycle of physiological signals is derived by above the peak detection, such as heart rate and so on. The following example is to introduce the algorithm computing ideas according to the heart rate.

The original data is set to be $\mathrm{y}=\mathrm{d}_{1}, \mathrm{~d}_{2}, \ldots, \mathrm{d}_{\mathrm{N}}$, , where $\mathrm{N}$ is the total number of the sampling data, $d_{i}$ represents the amplitude of the $i$-th number. The sampling frequency is $\mathrm{F}$, the total sampling time is $\mathrm{T}$.

$$
N=F \bullet T
$$

Data returns the location after peak detection algorithm where the number of vertices array is $\mathrm{P}, \mathrm{P}=\mathrm{s}_{1}, \mathrm{~s}_{2}, \ldots, \mathrm{s}_{\mathrm{m}}$. Wherein, $\mathrm{s}_{\mathrm{i}}$ is the $\mathrm{i}$-th extracted vertex number,, $\mathrm{m}$ is the number of extracted vertices. The calculated formula is can be drawn for heart rate HR:

$$
H R_{i}=\frac{F}{s_{i}-s_{i-1}}(1<i \leq m)
$$

\subsection{Outlier Detection}

The role of outlier detection algorithm is the removal peak before error extraction. Due to the presence of noise, peak detection results will be the actual vertex which is non peak, then there is the error. Assuming that the error peak $\mathrm{Sj}$ is extracted between the $\mathrm{Si}$, $\mathrm{Si}+1$ (as the error peak 4 between peak 3 and 5 is shown in Figure 4 (b) ), then

$$
\begin{gathered}
s_{i}<s_{j}<s_{i+1} \\
\Delta s=\min \left(s_{j}-s_{i}, s_{i+1}-s_{j}\right) \leq \frac{1}{2}\left(\left(s_{j}-s_{i}\right)+\left(s_{i+1}-s_{j}\right)\right)=\frac{1}{2}\left(s_{i+1}-s_{i}\right)
\end{gathered}
$$

$\mathrm{s}_{\mathrm{j}}$ heart rate $\mathrm{HR}_{\mathrm{j}}$ and $\mathrm{HR}_{\mathrm{i+1}}$ are calculated by the formula (2), an offset of them is bound to more 2 times than the normal value. This conclusion can assume that if the deviation size of heart rate is from the normal range in a certain range, the value is an outlier. The problem is transformed to the problem of outlier detection (Outlier Detection).

Aiming at this problem, a special algorithm is designed, it is Gauss Outlier detector. The algorithm assumes the data distribution is Gauss distribution $N\left(\mu, \sigma^{2}\right)$ in a short period of time. According to the Gauss distribution, it is combined with the actual needs, 
if the 3 standard deviation of the data is beyond $3 \sigma$, the data is as outliers, which constitute the core of the Outlier detection algorithm. The data is divided into continuous window, each window size is the length of 16 data, the time is about $8 \mathrm{~s}$. In addition, in order to ensure that the algorithm has more robustness, before Gauss distribution is used to calculation of abnormal value, a maximum value and a minimum value are removed within window data, but also the nearest distance value is removed to the detected value, experiments show that the algorithm can well identify outliers in the data. Figure 4 (b) shows the effect of outlier detection algorithm, it can be seen in Figure 4 (a) that abnormal values are correctly detected and removed.

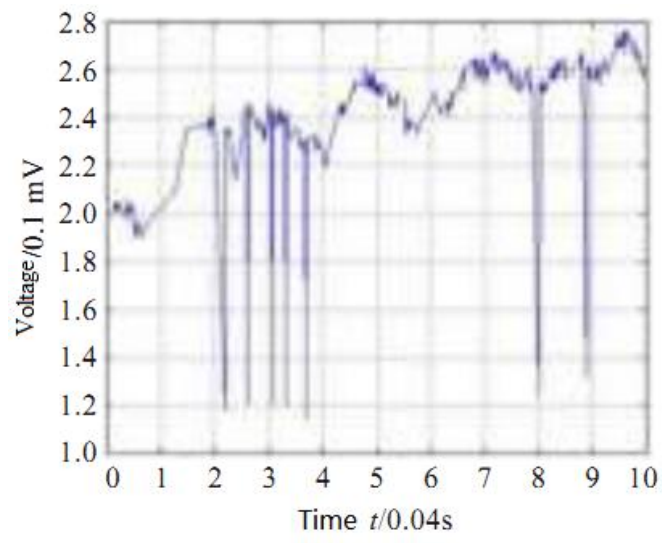

(a) The original heart rate variation

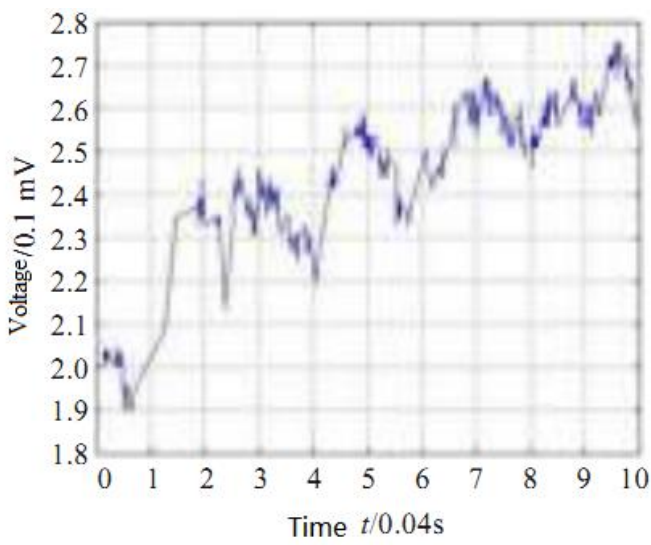

(b) The heart rate figure after Outlier detection

Figure 4. The Effect of Outlier Detection Algorithm

\section{Simulation and Performance Analysis}

\subsection{The Experimental Process}

In the whole process of the experiment, the experimenter will wear Berkeley Tricorder, the collected data types including ECG, EMG, Bio-impedance and 3 axis acceleration. The whole experiment was conducted for 5 times, data of 3 to 5 players are collected in each time, the experiment is completed to 5 times within 3 months, each experiment are arranged between two and five in the afternoon.

The experiment was divided into three stages, in the first stage, the experimenters do some basic preparation activities, such as footwork exercises, the height or distance ball exercises; in the second stage, the experimenter trains with the addition two participants; in third stage, the situation, the experimenter is 1 to 1 games with another participants at the same level. The average test time is about 7 to $8 \mathrm{~min}$. According to the arrangement, at the three stages, the exercise intensity of the first phase is minimum, the exercise intensity of the second phase is more strength than the first stage, the movement intensity at last match stage is no difference with the exercise intensity in 1 to 2 training stage.

All the subjects are from badminton professional students in sports department of Hunan International Economics University, there is a total of 12 subjects. There is a badminton coach in professional subjects, his age is 37; the remaining 11 per capita are for badminton professional students, their ages are 19 to 21 years old, including two badminton training for 8 years, remaining are in the 1 to 4 years. There are 6 experiments totally, some of which were involved in many times, data is collected to a total of 22 person / time, a total time of all the data is $4 \mathrm{~h} 38 \mathrm{~min}$. The problem of equipment off and 
bad contact are removed, effective data of ECG is 16 person / time, the effective EMG data is for the 19 people / times.

\subsection{The Difference of ECG in Different Stages of Exercise}

One of the coach concern important issues is whether the ECG levels of athletes have significant differences in basic training, strength training and competition stage. The results will be an important basis for coaches to judge the state of athletes. The average variation of the 16 / ECG is analyzed in the three stages, the result diagram is shown in Figure 5. The abscissa represent the three stages of the experiment, the vertical axis represents the average value of ECG, unit is times/s. The average value of ECG are Respectively 2.44, 2.71, 2.78 in the three stages. Paired-Sample T Test analysis showed that there are the significant differences of ECG in basic training, strength training and competition stage.

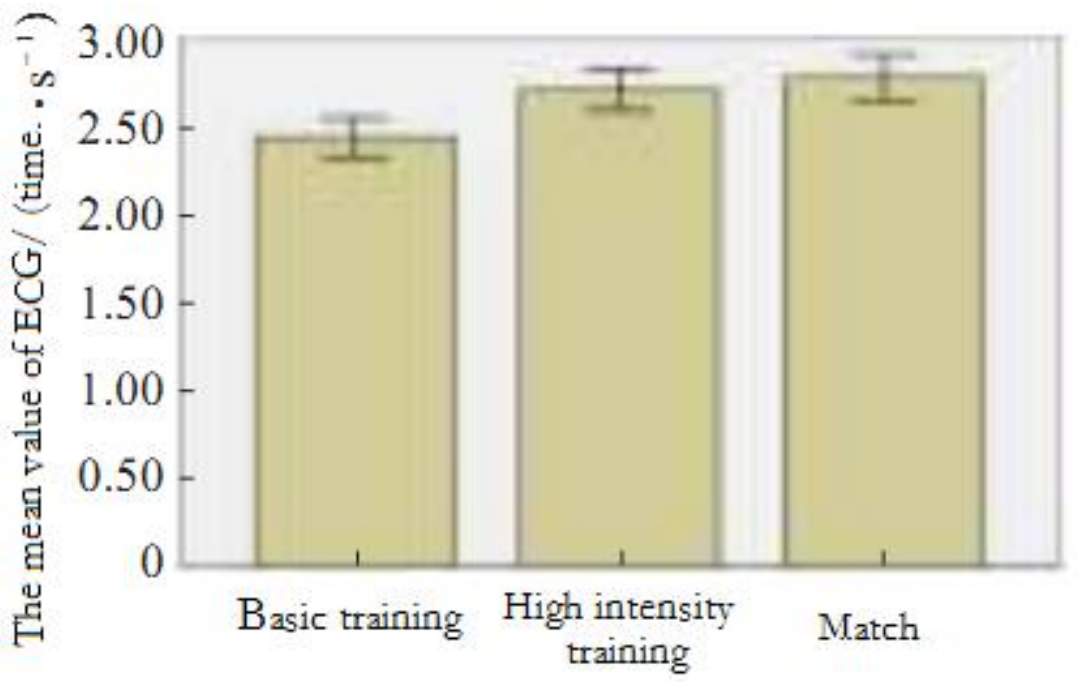

\section{Figure 5. The Average Change of ECG in the Three Stages}

In the three stage, the exercise intensity is larger in the great intensity training period than basic training phase, so the level of ECG was higher. According to the evaluation of coach, the sport strength of athletes in the intensive training are basically the same in the and competition, but it is judged from the results that the game player ECG level was significantly higher than that of high intensity training phase in ECG level. Thus it can be seen that players in the game stage have the level of tension, pressure is larger then training, so as to these reflect the changes in the level of ECG.

\subsection{The Same Player ECG Value Analysis with the Same Exercise Intensity}

Another important issue is whether athletes' ECG value is relatively stable under the same sports strength, which is concerned with the coaches. If the experiment can draw this conclusion, through the observation of the players in the ECG value, the coaches can determine whether the state of athletes is normal. If the ECG data of a player on the same day is significantly different from the normal level, the coach can understand by this information that state at that time and normal state of athletes is different, and it is as a basis for adjusting the training amount of athletes.

During the experiment, 5 people were involved in the experiment many times, wherein 3 people participated in the 3 experiment, 2 people participated in the 2 experiment. The 5 changes of ECG are shown in Figure 6. 


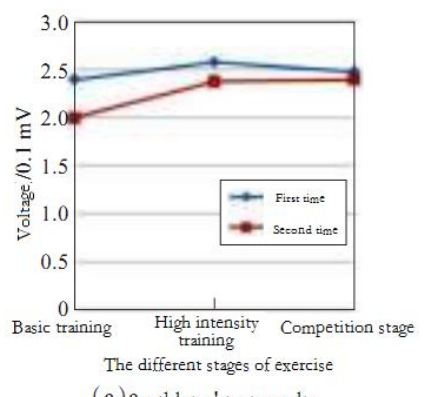

(a) a athletes' test results

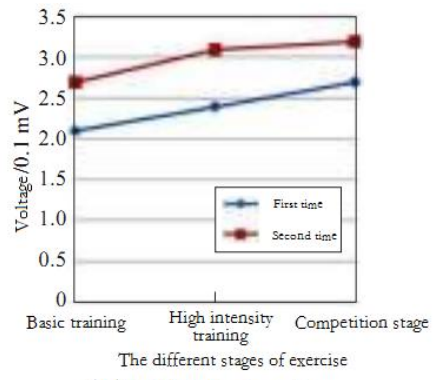

(b) b athletes' test results

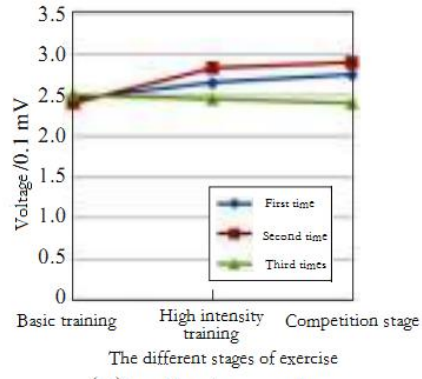

(c) c athletes' test results
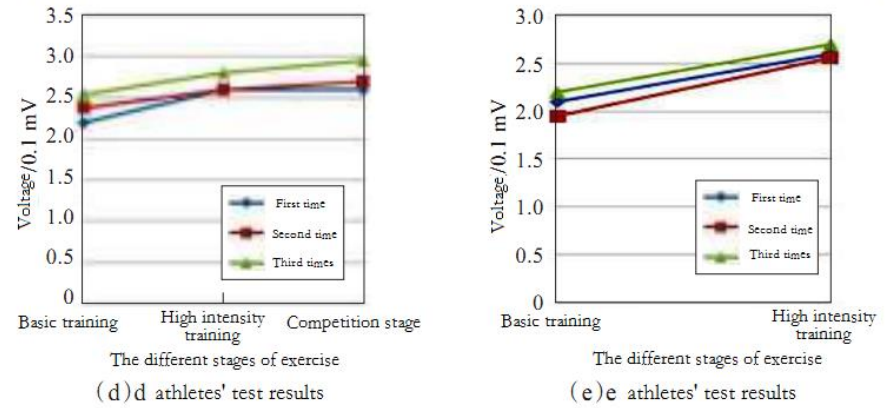

Figure 6. The Same Player ECG Changes In Different Date

Note: (a) (E) respectively the ECG changes with different dates for the 5 athletes, wherein the athletes were tested 2 times in Figure 6 (a) and (b), athletes were tested 3 times in Figure 6 (c) (e). As can be seen from the data, each player is measured at different times, their ECG is the same. The athletes have some differences in Figure 7, the ECG size and changes of the remaining 4 are the same. And ECG in Figure 6 (b) may be inconsistencies due to his state difference is relatively large in the two tests. It can be concluded from the results that the size and change of ECG are the same from the overall trend. Athletes' ECG is stable in the sports strength.

\subsection{ECG Variation of Different Level Athletes}

According to the understanding of the athletes, 5 athletes are selected and they are tested at different levels, and the changes of their ECG are observed, the experimental results shows in Figure 7.

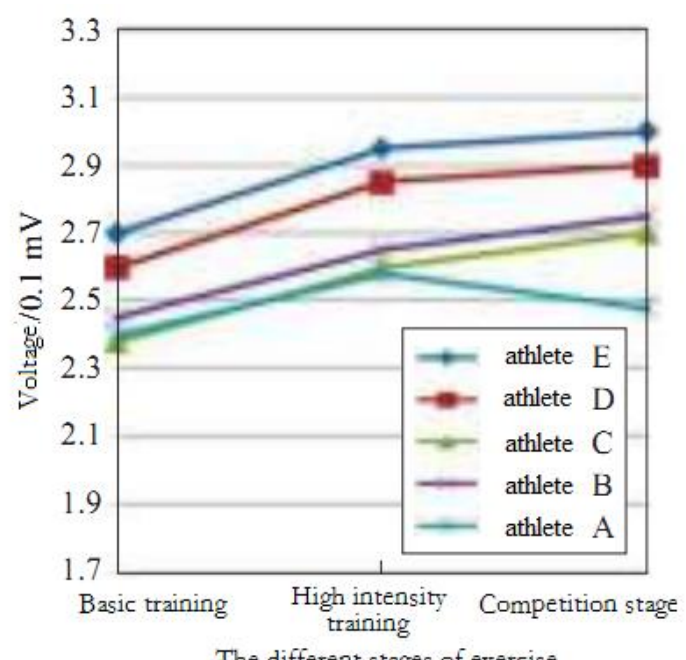

Figure 7. Comparison of Five Athletes' ECG Changes In Different Levels 
In Figure 7, the level of A athlete is the highest, it is followed by B athlete, and finally, athlete $\mathrm{E}$. In addition to the $\mathrm{C}$ player, the remaining 4 ECG size grade distribution is very obvious, the higher the level of the athletes, the lower their ECG. According to the analysis of the coach, ECG of athlete $\mathrm{C}$ is lower than that of B player, but athlete $\mathrm{C}$ technical level is no as athlete $\mathrm{B}$, because athlete $\mathrm{B}$ has been learning badminton athletes for 8 years, and $C$ player only has two years for study, so athlete $C$ is no better than athlete $\mathrm{B}$ from a technical level. However, the coach also pointed out that although the athlete $\mathrm{C}$ only has studied for two years, his progress is very rapid, athlete $\mathrm{C}$ is high level athlete in the same grade. Thus, it is concluded that there is a certain correlation with the magnitude of ECG between the level of the athletes. There is only a very preliminary results, the results need to be combined with the quality of athletes, for example, athletes are the strengthb type, the speed type, the skill type or etc.. From the coach feedback, under the different motion states, the ECG value is used as the reference index for the assessment of the athletes' level and the selection of athletes.

Co-relation discussion: Physiological Plan performed is used to real-time audience feedback, Figure 8 shows that the real-time audience feedback were tested in Physiological Plan. Figure 9 shows an experimenter in the wearable device.

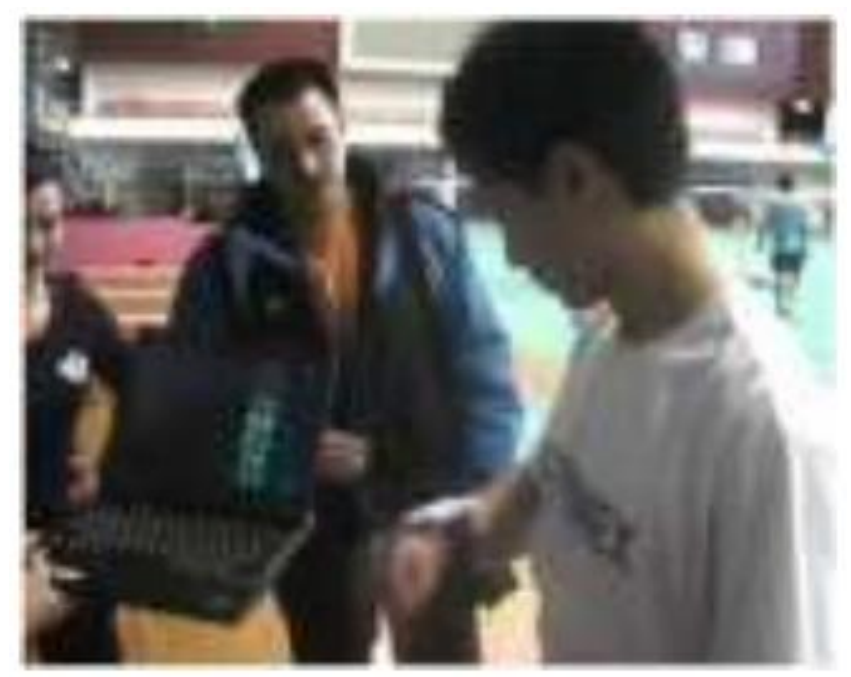

Figure 8. Real-Time Feedback Test

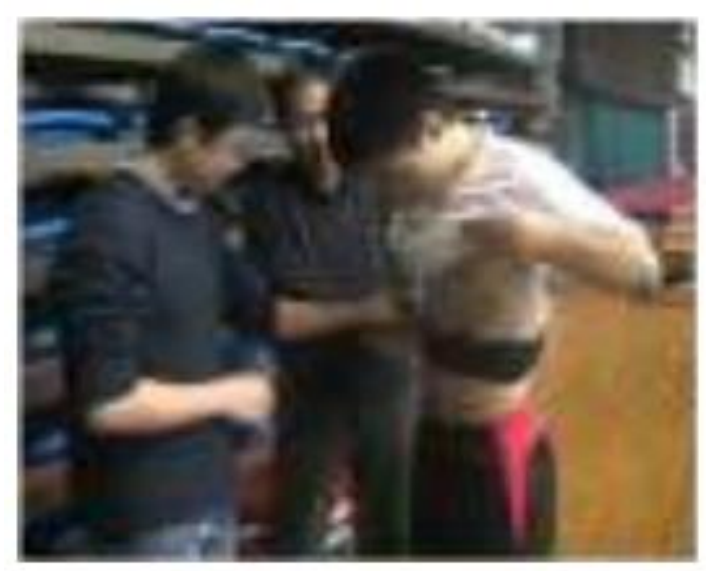

Figure 9. Testers Are Wearing Sensor

Gathering Physiological Data: The ProComp+ A-D converter can receive signals, sampling data at $256 \mathrm{samples} / \mathrm{sec}$, from a number of sensors, it can be embedded in 
normal clothing. We will use the ECG sensors for our purposes. Electrocardiogram (ECG) Heart Rate Variability (HRV) Spectrum Analysis is obtained from the heart to create Physiological Models.

ECG Heart Rate Variability: Heart rate variability (HRV) can be found by taking the time between an R-R peak interval, the increased HRV has been correlated with increased stress levels. Figure 10 is a normal ECG waveform

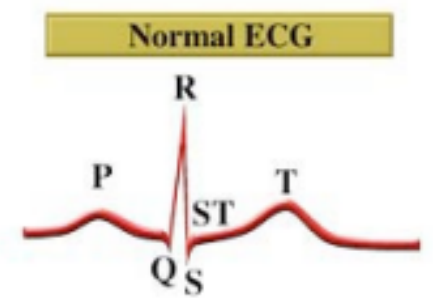

Figure 10. A Normal ECG Waveform

ECG HRV Spectrum Analysis: A fourier transform of the ECG HRV data was taken to obtain a power spectral density graph. It has been known that the based increased frustration shows an increase in the lower frequency band, whereas the increased appreciation (similar to concentration) shows an increase in the medium frequency band.

The basic training and high intensity training phase are compared, their $\mathrm{P}$ values were less than 0.0001 . There is $\mathrm{P}$ value of 0.006 in high intensity training and competition stage. There is a significant difference between the two.

This work is studied on the basis of previous research (Fairclough, 2009). One of the further work is to improve the existing equipment, the athletes are made more comfortable and more convenient. Another important work is to collect more data, more data is analyed, the change law of corresponding to ECG, EMG is research for different motion.

\section{Conclusions and Outlook}

According to the physiological data, the athletes' state is sensed and analyzed real-time, and the intensity of movement training is controlled and tactics is developed based on these data, these are the key problem which is concerned by coaches. The existing problems are investigated and analyzed in the badminton training, Psychological Plan has been researched and designed in this study, a interactive training analysis system is designed based on the physiology calculation. The system includes the hardware layer, data layer, algorithm layer and interface layer. Berkeley Tricorder platform is used as the hardware layer; data of every sensor data will be unified through the adapter pattern in processing layer; algorithm layer includes filtering algorithm, peak detection algorithm and Outlier detection algorithm; interface layer is interact with the coach, and the results show to the coach. The electrocardiogram (ECG), electromyogram (EMG) and 3D acceleration of the athletes and other information can be collected and analyzed in the system and the data analysis and processing results are back to the coach, coaches are helped to analyze athletes' state. Experiments show that the Physiological Plan system can effectively help the coaches to monitor and analyze the states of players, the real-time data of athletes are obtained in training and competition. At the same time, the Physiological Plan system data can help the coach to analyze the athlete's mental state, it also has an important guiding role in the assessment of the players' ability and material.

Although the equipment is still in the initial stage, the coach and the players showed great enthusiasm to test it, and some problems are found in equipment, but also the many useful suggestions are given. The belt is used in original equipment, but it is found that test equipment is easy to fall off in the athletes such violent activities, the dress form is 
improved later, but also the comfort is improved. But there are several problems as follows, such as clothes can change size, which can not be suitable for every athlete, equipment needs to be cleaned after use, and now to clean up, the sensors need to be removed, it is not convenient.

\section{Acknowledgments}

This study is sponsored by the Scientific Research Project (NO. 14A084) of Hunan Provincial Education Department, China.

\section{References}

[1] Fairclough, S.H.,Fundamentals of physiological computing. Inter. Comp., 2009, 21: 133-145.

[2] Allanson, J. and S.H.A. Fairclough, Research agenda for physiological computing. Int. Comp., 2004, 16: 857-878.

[3] Malmo, R.B. and H.P. Malmo, On electromyographic (EMG) gradients and movement-related brain activity: significance for motor control, cognitive functions and certain psychopathologies. Int. J. Psychophysiol., 2000, 38: 143-207.

[4] Saponas, T.S., D.S. Tan and D. Morris, Demonstrating the feasibility of using forearm electromyography for muscle-computer interfaces. Proceedings of the SIGCHI Conference on Human Factors in Computing Systems, Florence, Italy, April 5-10, 2008, pp: 515-524.

[5] Cacioppo, J.T., L.K. Bush and L.G. Tassinary, Microexpressive facial actions as a function of affective stimuli: Replication and extension. Personality Soc. Psychol. Bull., 1990.,18: 515-526.

[6] Porges, S.W. and E.A. Byrne, Research methods for measurement of heart rate and respiration. Biol. Psycho., 1992, 34: 93-130.

[7] Calvert, S.L. and S.L. Tan, Impact of virtual reality on young adults physiological arousal and aggressive thoughts: Interaction versus observation. J. Applied Dev. Psycho., 1994.,15: 125-139.

[8] Blascovich, J., W.B. Mendes, S.B. Hunter and K. Salomon, Social facilitation as challenge and threat. J. Personality Soc. Psychol., 1999, 77: 68-77.

[9] Hartmann, B., L. Abdulla, M. Mittal and S.R. Klemmer, Authoring sensor-based interactions by demonstration with direct manipulation and pattern recognition. Proceedings of the SIGCHI Conference on Human Factors in Computing Systems, April 30-May 3, 2007, San Jose, CA., USA., pp: 145-154.

[10] Ashbrook, D., MAGIC: A motion gesture design tool. Proceedings of the SIGCHI Conference on Human Factors in Computing Systems, April 10-15, 2010, Atlanta, GA., USA., pp: 2159-2168.

[11] Arroyo, E., S. Sullivan and T. Selker, Car coach: A polite and effective driving coach. Proceedings of the Extended Abstracts on Human Factors in Computing, April 22-27, 2006, Montreal, Canada, pp: $357-$ 362.

[12] Fogarty, J., S.E. Hudson, C.G. Atkeson, D. Avrahami and J. Forlizzi, Predicting human interruptibility with sensors. Trans. Comput. Hum Interact, 2005., 12; 119-146.

[13] Kientz, J.A., S. Boring, G.D. Abowd and G.R. Hayes, Abaris: Evaluating Automated Capture Applied to Structured Autism Interventions. In: UbiComp 2005: Ubiquitous Computing, Beigl, M., S. Intille, J. Rekimoto and H. Tokuda (Eds.). Springer Berlin Heidelberg, USA., ISBN: 13-9783540287605, pp: 323339.

[14] Bachlin, M., K. Forster and G. Troster, SwimMaster: A wearable assistant for swimmer. Proceedings of the 11th International Conference on Ubiquitous Computing, September 30-October 3, 2009, Orlando, Florida, USA., pp: 215-224.

[15] Chang, K.H., M.Y. Chen and J. Canny, Tracking Free-Weight Exercises. In: UbiComp 2007: Ubiquitous Computing, Krumm, J., G.D. Abowd, A. Seneviratne and T. Strang (Ed.). Springer Berlin Heidelberg, USA., ISBN: 9783540748526, pp: 19-37.

[16] Michahelles, F. and B. Schiele, Sensing and monitoring professional skiers. IEEE Pervasive Comput., 2005., 4: 40-46.

[17] Michael, B., K. Bernhard and L. Martin, Computerized real-time analysis of football games. IEEE Pervasive Comp., 2005,4: 33-39.

[18] Naima, R. and J. Canny, The Berkeley tricorder: ambulatory health monitoring. Proceedings of the 6th International Workshop on Wearable and Implantable Body Sensor Networks, June 3-5, 2009, Berkeley, CA., pp: 53-58. 

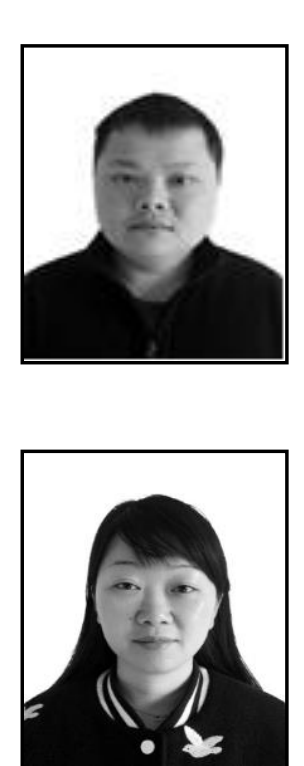

\section{Authors}

Ding Xiong, he received the bachelor's degree in Computer science and technology from Hunan University of Science and Technology, china, in 2003.He received him Master's degree in Computer science and technology from Hunan University, china, in 2010. He is currently working as Senior Engineer at the Information science and technology, Hunan International Economics University. Him research interests include network computing and Algorithm application.

Lu Yan, she received the bachelor's degree in Computer science and technology from Hunan Normal University, china, in 2002. She received her Master's degree in Technical economics and management from Central South University, china, in 2010. She is currently working as Senior Engineer at the Information science and technology, Hunan International Economics University. Her research interests include Algorithm application and distributed computing. 
International Journal of $u-$ and e- Service, Science and Technology Vol. 10, No.3 (2017) 